\title{
Déjouer le temps et l'espace, reprendre du pouvoir
}

Marie-Pierre Julien, Olivier Wathelet et Lucie Dupré

\section{(2) OpenEdition}

Journals

Édition électronique

URL : https://journals.openedition.org/tc/8802

DOI : $10.4000 /$ tc. 8802

ISSN : 1952-420X

Éditeur

Éditions de l'EHESS

Édition imprimée

Date de publication : 17 mai 2018

Pagination : $12-27$

ISBN : 978-2-7132-2750-9

ISSN : 0248-6016

Référence électronique

Marie-Pierre Julien, Olivier Wathelet et Lucie Dupré, « Déjouer le temps et l'espace, reprendre du pouvoir », Techniques \& Culture [En ligne], 69 | 2018, mis en ligne le 17 mai 2020, consulté le 29 septembre 2022. URL : http://journals.openedition.org/tc/8802 ; DOI : https://doi.org/10.4000/tc.8802 


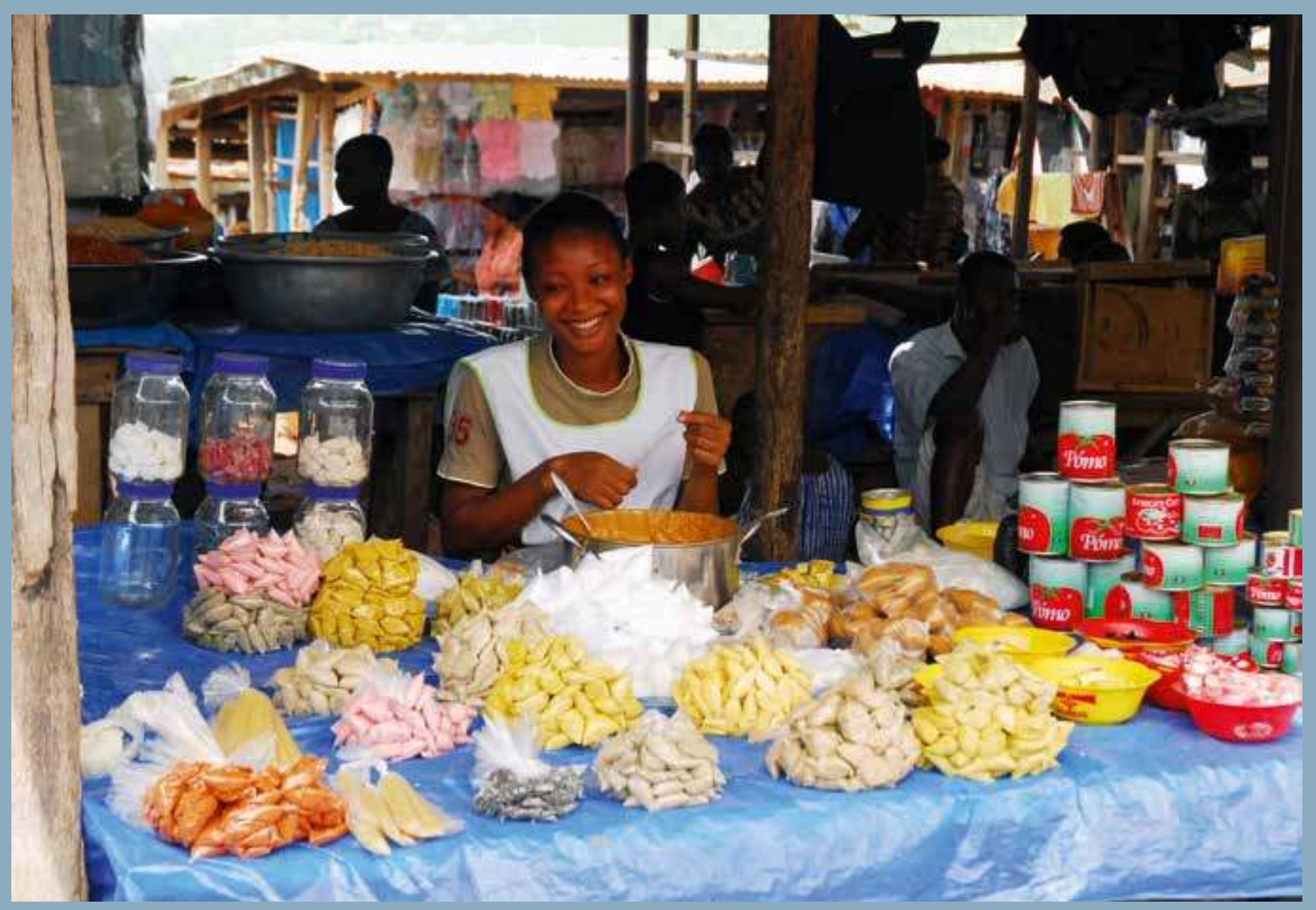




\section{Déjouer le temps et l'espace, reprendre du pouvoir}

Appréhendée à des échelles diverses et traversée par des enjeux qui vont de la sécurité («nourrir le monde ») à la souveraineté alimentaires (droit des peuples à définir leurs politique et pratiques alimentaires) en passant par les arbitrages quotidiens et domestiques (que faire à manger ce soir?), la consommation relève clairement d'enjeux géopolitiques étudiés au niveau des formes de production et de consommation - de l'achat à l'ingestion à proprement parler. Cette double focale, évidente en apparence, tend cependant à éclipser le rôle de la conservation ${ }^{1}$ des aliments. Par contraste avec les recherches récentes consacrées à l'alimentation, ce «moment» de la chaîne alimentaire apparaît doublement sous-exploré: d'une part par le faible nombre de travaux qu'il suscite, même si des anthropologues de renom s'en sont préoccupés, comme Claude Levi-Strauss (1958) analysant le cru et le cuit dans une perspective structuraliste, Jack Goody (1985) resituant les pratiques culinaires dans leurs contextes historiques et socioéconomiques ou encore Alain Testart (1982) qui s'est appuyé sur l'analyse des formes de stockage alimentaire pour remettre en question l'opposition entre société de chasseurs-cueilleurs et société agricole. D'autre part, à ces quelques exceptions près, l'approche réservée à la conservation alimentaire tend à la réduire à ses aspects «techniques», en tant que procédé permettant de soustraire les aliments aux processus de leur dégradation naturelle. De ce fait, la conservation des aliments semble confinée au registre des processus techniques dont on ne parle qu'en contrepoint de sujets plus importants, comme si elle n'était associée à aucune forme d'enjeux propres. Au point que nous pourrions faire l'hypothèse qu'elle relèverait du temps des infrastructures (Karasti et al. 2010) dont les mutations, plus lentes que celles de la temporalité des «projets», semblent échapper à la perception des usagers et de leurs analystes.

Pourtant, et c'est bien l'objectif de ce volume que d'en faire la démonstration, la conservation ne peut se réduire à un moment d'attente entre la production ou l'achat et la consommation alimentaires. Il ne s'agit pas simplement de préserver durablement les propriétés organoleptiques, 
nutritionnelles et sanitaires d'un produit. La conservation est un sujet en tant que tel et, à ce titre, elle rend compte de choix de société et de choix politiques à part entière. Dès lors, tout comme les travaux conduits à l'échelle de systèmes d'approvisionnement alimentaire (Fine \& Leopold 1993, Mintz 1985) ont montré l'intérêt d'analyser ensemble production-distribution-consommation, la conservation est elle aussi irréductiblement liée à ces trois dimensions.

Notre réflexion collective s'est appuyée sur deux journées de présentations, d'échanges et de débats entre les contributeurs de ce volume². Ce temps fort a été suivi d'un travail éditorial, tout aussi intense bien que conduit à distance, avec chacun des auteurs que nous remercions ici chaleureusement pour leur enthousiasme et leur contribution à l'édifice commun. Ces échanges nous ont conduits à adopter un double parti pris: d'une part, observer comment les pratiques de conservation (industrielles, artisanales et domestiques) se complètent plutôt qu'elles ne s'opposent, et d'autre part, en accord avec l'anthropologie des techniques, montrer comment celles-ci donnent à voir un monde singulier fait de pouvoirs, de préférences, de contraintes, de possibilités mises en tension. Cette intention de travail a donc fait tenir ensemble les contributions réunies dans ce volume: montrer que les techniques de conservation, aussi diverses soient-elles, ne sont pas seulement « des actes traditionnels efficaces» (Mauss 1934), mais renvoient à une façon d'organiser les rapports sociaux, de définir ce qui importe, d'attribuer une valeur à l'aliment comme à ceux qui le mangeront, en lien avec un milieu ou un environnement spécifiques dotés de contraintes propres.

Dès lors, nous proposons d'interroger l'évidence: pourquoi conserver les aliments? Pour leur donner du temps et rendre possible leur consommation ultérieure? Mais la conservation ne fait-elle que conserver? Autant de questions qui nous invitent, dans le prolongement des réflexions ouvertes par le concept de société de consommation, à questionner la «société de conservation » comme grille de lecture pour repérer les tensions et les enjeux qui structurent la question alimentaire contemporaine et passée. Pour en rendre compte, les contributions réunies ici permettent d'explorer deux dimensions étroitement imbriquées mais qu'on isole pour les commodités de l'analyse. La première insistera avant tout sur les conséquences temporelles de la conservation (accumuler, bonifier, capitaliser) et la seconde développera les conséquences de la circulation des denrées conservées dans l'espace (faire circuler, partager, échanger).

\section{La société de conservation entre production et consommation}

Les techniques de conservation des produits alimentaires, en tant que moyen de maîtriser le temps, ont fait partie du processus d'humanisation (Testart 1982, Kujit \& Finlayson 2009). Avant l'agriculture et l'élevage, le stockage des aliments collectés ou chassés a ainsi été la seule possibilité de mettre un groupe à l'abri de la famine. Les modes de conservation évoluent donc avec les changements des modes de production/extraction des ressources alimentaires, et la façon de les faire circuler au sein d'espaces de plus ou moins grande ampleur. La société de 
consommation est une société dite d'abondance - du moins en apparence ou du point de vue idéologique - comme l'a analysé l'école de Francfort dès les années 1950 ou, en France, des auteurs comme Baudrillard et ses successeurs dans les années 1970 : liée à une production de masse relayée par des techniques de conservation nouvelles et décisives. À cet égard, les technologies modernes montrent une grande diversité dans l'art de conserver: chaîne du froid, appertisation, sous vide, UHT, dessiccation, affinage, agents conservateurs naturels ou chimiques, micro-organismes, nanoparticules, etc., et dans les infrastructures afférentes (chambres froides et frigorifiques, entrepôts, conteneurs, etc.). Dans une certaine mesure, elles répliquent au sein du commerce mondial les saloirs, caves, glacières, greniers et autres lieux de stockage et de conservation domestiques que l'on retrouve en différents points du globe.

Les grandes et moyennes surfaces consubstantielles de la société de consommation ont, elles aussi, dû et pu compter avec l'évolution des techniques de conservation (Cochoy 2010, 2017). Conséquemment, la consommation s'en est trouvée, elle aussi, fortement transformée: massification, diversification de l'alimentation, désir d'exotique (Régnier 2004). En effet, autour de quelques technologies clefs (conserves, chaînes du froid industrielle et domestique, boîtes à conserver et à transporter...), les liens entre cuisine et approvisionnement ont eux aussi subi d'importantes transformations. Les réfrigérateurs et congélateurs occupent une place particulière dans cette histoire: sorte de représentant idéal d'une modernité qui confinerait au gaspillage. Les «oubliés des fonds de frigo », pour reprendre le nom d'un projet de design canadien ${ }^{3}$ dédié à lutter contre le gaspillage, illustrent bien l'idée d'un lieu d'opulence, où les surplus s'accumulent avant de périmer en silence derrière les portes opaques, alors que de plus en plus d'hommes et de femmes ne mangent pas à leur faim ${ }^{4}$. Entre autres exemples d'initiative récente, les «frigos de rue» cristallisent ainsi les pratiques de «partages solidaires» comme un moyen de rendre disponibles «à tous ${ }^{5}$ des produits conservés non pas dans des lieux privés mais au cœur de l'espace public. C'est aux frigos individualisés qui peuplent les maisons, que Gaël David et Antoine Veteau, respectivement photographe et journaliste, se sont intéressés. Dans un webdocumentaire initié à partir d'une réflexion sur le pouvoir d'achat, ils ont dressé le portrait et interrogé le contenu des frigos d'une dizaine de personnes, qui ont accepté, non sans réticence parfois, de leur en ouvrir la porte. Car le frigo, et son extension le congélateur, touchent à l'intimité et les montrer implique le risque de prêter le flanc au jugement d'autrui. Contre l'idée d'un appauvrissement de l'expérience (Howes 1990), ces «boîtes à froid » génèrent au contraire de la singularité et de la richesse émotionnelle et sensorielle. Camille Adamiec, épaulée par la dessinatrice Marina Savani, expliquent la façon dont les congélateurs permettent à leur propriétaire de négocier leur rapport au temps microsocial de la famille comme à celui macrosocial de la saisonnalité, en conservant les trésors alimentaires (herbes, viandes ou plat préparés) ou non-alimentaires (corps du hamster mort ou bonhomme de neige). 


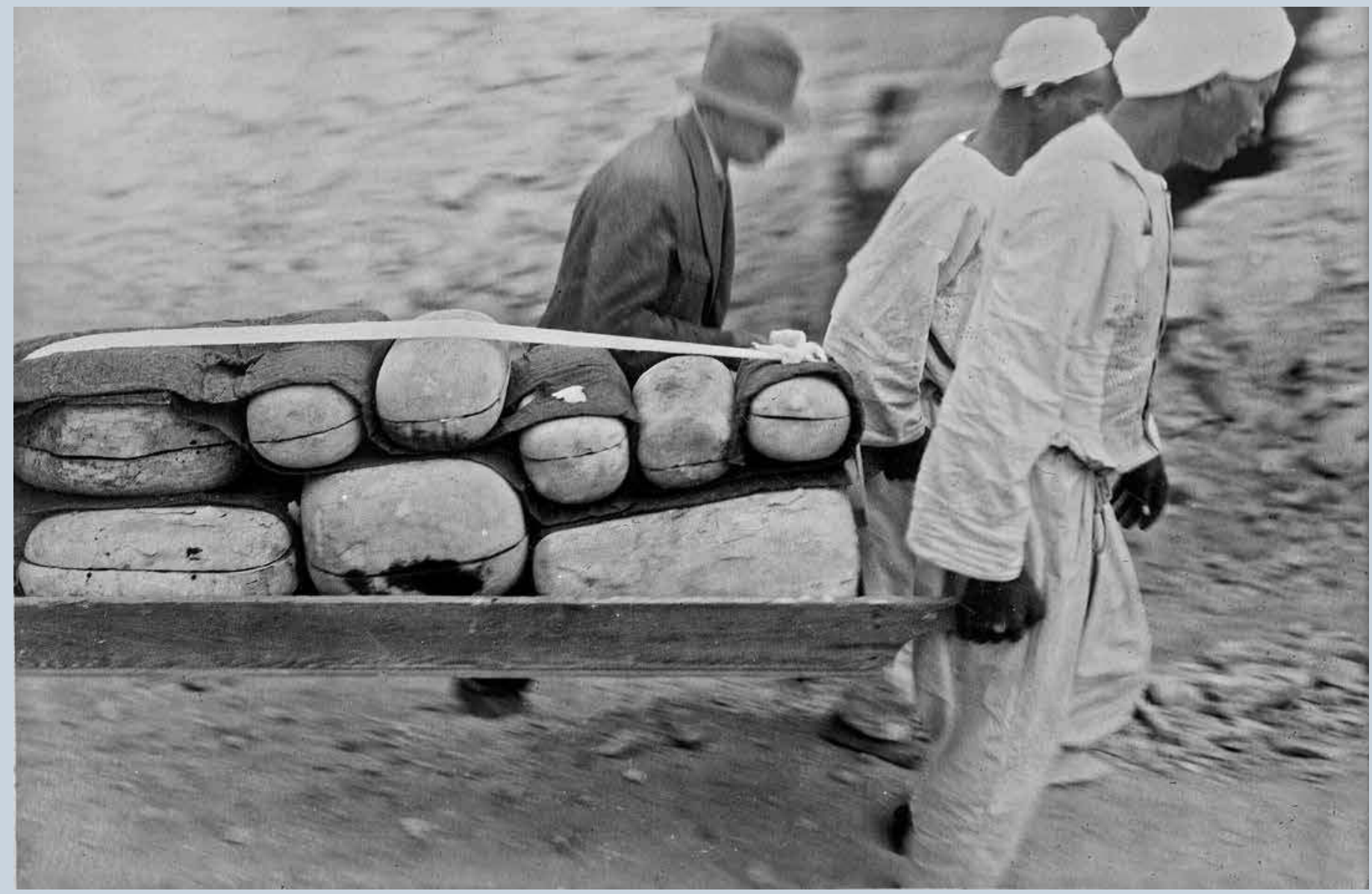

Découverte du tombeau de Toutankhamon

Boîtes ovales contenant de la nourriture momifiée, gigots de moutons, etc. 


\section{Accumuler, bonifier, capitaliser}

Conserver, c'est donner du temps à l'aliment, ce qui peut être une façon d'accroître sa valeur, en l'accumulant, en le retenant ou en le transformant. Ce sont ces liens entre accumulation, bonification et capitalisation que le premier ensemble de textes de ce volume explore à travers des approches disciplinaires et analytiques différentes. Cette valorisation, passe par deux moyens: la fabrication d'un goût spécifique, socialement recherché, et le jeu de la rareté. Les produits dérivés du lait, dont le fromage et le beurre - qui sont en soi des moyens de conserver le lait cru - illustrent particulièrement bien cette problématique. Trois articles portent sur ces produits qui mettent en évidence la complexité de ces liens entre accumulation, bonification et capitalisation. En détaillant la chaîne opératoire de la transformation du lait en beurre rance dans la Mongolie contemporaine, Sandrine Ruhlmann montre l'importance sociale du stockage de produits ruraux dans cette société désormais très urbanisée. Ainsi, elle éclaire la construction sociale d'un goût qui peut être amené à changer au fur et à mesure du vieillissement du beurre - faisant du reste bien comprendre à quel point les normes en matière de conservation ne se valent pas -, n'est pas l'objet d'une capitalisation comme c'est le cas pour le fromage en Europe. À l'inverse, Katia Fersing analyse la façon dont, avec la maîtrise de l'industrialisation du processus de transformation du pain de caillé en fromage dit de Roquefort, les distributeurs ont pris le contrôle économique de la production et de sa capitalisation. Ils ont travaillé à mettre en adéquation le goût du fromage vendu avec celui socialement acceptable par les consommateurs urbains, et surtout ont participé à définir une AOP afin de se différencier des caves voisines et faire capitaliser leur terroir (Boltanski \& Esquerre 2017). S'intéressant également au fromage de lait de brebis et aux enjeux mis en évidence par la création d'une AOC, Valeria Siniscalchi révèle les relations conflictuelles entre les producteurs bergers sardes de Fiore Sardo, les producteurs industriels et les instances politiques régionales. Le goût, ses liens avec la biodiversité (des micro-organismes facteurs de la transformation) et la question de la pasteurisation du lait, occupent le cœur des tensions entre bonification et capitalisation. Lauteure montre ainsi le rôle des acteurs extérieurs à la Sardaigne dans ces conflits sardes. Elle considère à la fois l'action économique des distributeurs des Pouilles (région d'Italie qui commercialise la plus grande partie de la production) qui fixent le prix du fromage, et celle plus politique des «sentinelles Slow Food ${ }^{6}$ qui interviennent en soutien des producteurs bergers par la commercialisation de leurs fromages avant que leur qualité ne se dégrade. La maîtrise du temps est ici déterminante: il s'agit d'en donner, mais juste ce qu'il faut pour augmenter la valeur du fromage. Dans ces trois contributions, le goût, comme « construit social», est au centre d'enjeux politiques et économiques qui articulent accumulation, bonification et capitalisation des fromages européens.

Contre une certaine idée de sens commun, le jeu entre bonification et capitalisation bien étudié en Europe sur ce type de produit, est également à l'œuvre dans d'autres systèmes techniques, et notamment celui concernant l'industrie de la potasse en pays Dogon (Mali). 
Laurence Douny s'intéresse à l'efficacité de cette technique qui ne tient pas uniquement à ses capacités de conservation du mil mais, également, à son goût qui la rend nécessaire dans les recettes d'assaisonnement, à ses qualités thérapeutiques ou de neutralisation des sorts. Partant d'une description minutieuse de la chaîne opératoire qui permet d'obtenir la fameuse potasse, l'auteure montre qu'il n'y a pas toujours une claire délimitation entre bonification et capitalisation, car chez les Dogons, savoir utiliser la potasse permet de conserver des denrées et ainsi de protéger sa famille de la faim, mais, également, de maîtriser un goût socialement valorisé et de contenir la maladie et les sorts.

Les archéologues Jacob Morales, Pedro Henríquez-Valido, Marco Moreno-Benítez, Yurena Naranjo-Mayor et Amelia Rodríguez-Rodríguez ont pu mesurer la présence récurrente de feuilles de laurier dans l'analyse des restes trouvés dans des silos creusés au sein de grottes naturelles situées à 250 mètres d'altitude dans des falaises de Grande Canarie par des membres de la société agraire pré-hispanique de la première moitié de notre ère. Leur analyse chimique montre que les feuilles de laurier ont limité les ravages des insectes, ont eu un fort effet fongicide et ont inhibé la germination des graines. De la même façon qu'avec le roquefort, les fraîches cavités naturelles des falaises constituent des caches idéales pour protéger les stocks des razzias de concurrents (humain, animal, fongique...). Les auteurs mettent en évidence une lutte sans cesse renouvelée qui interroge les pratiques de l'industrie chimique au regard de ces procédés traditionnels.

Isabelle Bianquis et Isabella Borissova s'intéressent, en Yakoutie, à la dégustation de viandes et de poissons crus congelés, véritables délicatesses tant dans les cuisines populaires rurales que dans les restaurants huppés urbains. Alors que certaines techniques de conservation mettent l'accent sur la transformation de l'aliment pour lui donner un goût culturellement acceptable, le cru congelé des Yakoutes valorise le rapprochement avec ce qui est considéré comme «naturel ». La congélation permet ici la bonification du poisson qui, cru, répugne les mangeurs Yakoutes. Dans ce cas encore, la bonification s'articule à la capitalisation : la congélation permet de présenter dans des restaurants gastronomiques urbains une cuisine qui met en avant ses racines rurales, réputées proches de la nature et de ses bienfaits, politiquement valorisées dans une région postsoviétique.

Dans ces derniers exemples, la conservation apparaît comme une façon d'augmenter, par la transformation de son goût, la valeur d'un aliment. Elle revient alors à maîtriser l'évolution naturelle d'un produit vivant, pour lui conférer une plus-value économique et sociale. Mais il est parfois nécessaire, au contraire, de garder à l'identique certains aliments, d'assurer l'intégrité et la stabilité nutritionnelle qui leur confère leurs valeurs, et sans laquelle ils s'altéreraient. Samuel Pinaud analyse ainsi l'économie politique de la poudre de lait en Afrique de l'Ouest. L'auteur a mené une enquête à Bamako, au Mali, s'intéressant à la façon dont la conservation de la poudre de lait confère à ses metteurs en marché et revendeurs un double pouvoir: voyager dans le temps et voyager au loin. Ces deux pouvoirs cumulés placent cette denrée au cœur d'une économie laitière internationale dont Samuel Pinaud rappelle qu'elle s'ancre, sous couvert d'une action humanitaire, dans le développement et la reproduction de 


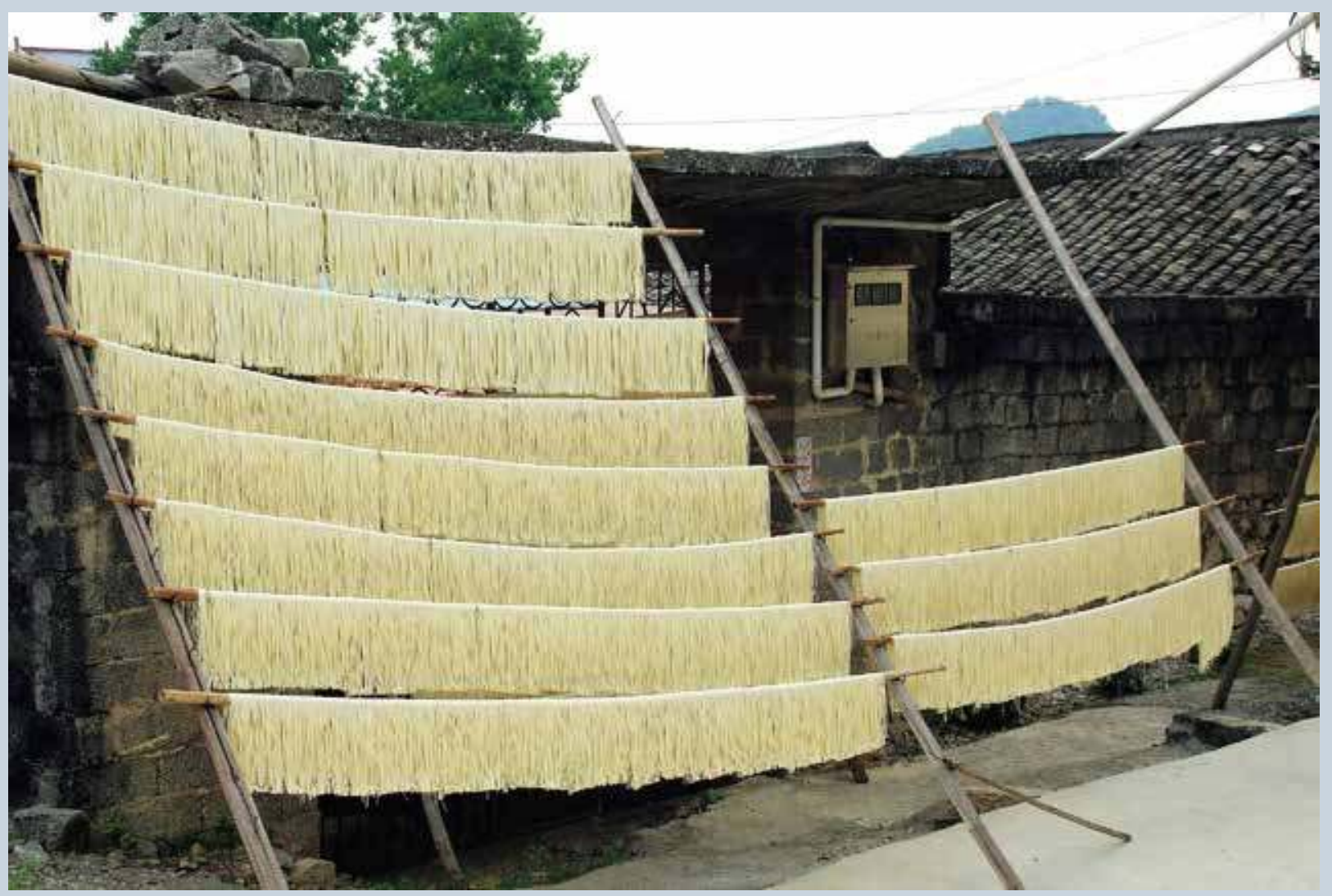

\section{Séchoir domestique de nouilles (Guilin, Chine)}

Le traitement préparatoire à la conservation des aliments oscille entre productions industrielles et pratiques domestiques, sans que les deux sphères soient toujours clairement distinctes. Par leur ampleur, les préparations à usage personnel peuvent parfois prendre des quantités et des formes impressionnantes, surtout lorsque la consommation de plusieurs semaines, voire d'une année, est pré-cuisinée et stockée en une seule fois. 
relations coloniales entre la France et le Mali aujourd'hui, à travers la conquête d'un marché économique que permet la (bonne) conservation de la poudre de lait.

La nécessité de conserver loin dans le temps, tout en continuant à veiller à l'intégrité des aliments, a constitué une préoccupation d'autant plus décisive que les sociétés étaient étroitement dépendantes des ressources de leurs environnements. Il fallait alors accumuler, stocker massivement des ressources souvent disponibles de façon très fluctuantes. Les archéozoologues Marie-Cécile Soulier et Sandrine Costamagno analysent les techniques des chasseurs-cueilleurs paléolithiques, entre - 80000 à - 14000 , pour constituer, stocker et conserver de grandes quantités de viande: les migrations automnales des rennes offraient d'abondantes ressources de qualité. Chasses collectives, abattages massifs, préparation de la viande selon différentes techniques - parfois assez sophistiquées - selon la consommation qui en sera faite. L'étude des os du gibier constitue cet indice ténu qui permet d'essayer de comprendre l'organisation et les techniques de conservation de populations du Magdalénien. Car les techniques de découpe sont un aspect important de la conservation comme le montre également Nicolas Lacombe à propos de l'évolution des marchés des viandes de lait dans deux îles méditerranéennes, la Corse et la Sardaigne. L'auteur montre la façon dont la découpe, le froid, l'emballage, participent à renouveler le marché et permettent de répondre à l'évolution des pratiques de consommation de cette viande de lait, historiquement très saisonnières (Noël et Pâques). Pour développer les débouchés et répondre aux nouvelles formes et saisons de consommation, les carcasses vendues entières lors des fêtes familiales traditionnelles sont désormais aussi découpées, emballées voire congelées, et permettent d'accéder aux consommateurs urbains et estivaux.

Conserver permet enfin de déplacer les biens, et de transformer radicalement la valeur d'usage de produits autrement ordinaires. Avec Pascal Lièvre, Géraldine Rix-Lièvre et Michel Récopé, nous partons au cœur d'expéditions polaires. La question alimentaire est une clef de la réussite - ou de l'échec - de ces mobilités extrêmes. Comment prévoir sa nourriture dans des situations aussi contraignantes? Et comment calculer la valeur précise de chaque aliment pour un corps à ce point contraint? Le climat, l'effort, les exigences de poids, de volume disponible qui contraignent l'espace-temps de la randonnée mais, aussi, l'expérimentation de la possibilité même de conserver, le plaisir de se restaurer, les changements de goûts et de dégoûts de chacun des participants dans cette situation extrême... Les auteurs rappellent combien le social et la technique se co-construisent dans les gestes, les corps et les émotions de chaque randonneur, donnant lieu à la constitution de différentes logiques d'expédition qu'ils identifient, au terme d'une observation pleinement participante. 


\section{Faire circuler, partager, échanger}

Sans être nécessairement contradictoire avec les logiques de capitalisation, la conservation permet également un échange, marchand ou non, et un partage de nourritures. Mais comment partager des aliments sans partager les savoir-faire qui vont avec leur conservation, leur préparation, leur consommation? Le cas de la morue décrit par Gérard Allemandou est à ce titre exemplaire. À la fois aliment transformé pour faciliter sa circulation, la morue est aussi un produit dont le succès dépend de la valeur accordée aux différentes recettes qui en ont permis la préparation, à travers les siècles. La mise en stock du capital cognitif est le pendant nécessaire - mais non suffisant - du succès de cet aliment transformé. Ce processus de transmission à double flux concerne tout autant les préparations contemporaines, à l'instar du Kéfir étudié par Renaud Debaillly, Christophe Lavelle et Émilien Schultz. $\mathrm{Au}$ contraire de la morue, la conservation de cette boisson ne tient pas à la préparation de l'aliment, mais à la capacité de conserver «vivant» les grains et de les transmettre - gratuitement ou dans le cadre d'une relation marchande via notamment les circuits sur Internet. Chaque participant à ces échanges doit de son côté réunir les connaissances utiles et les ingrédients stockés pour

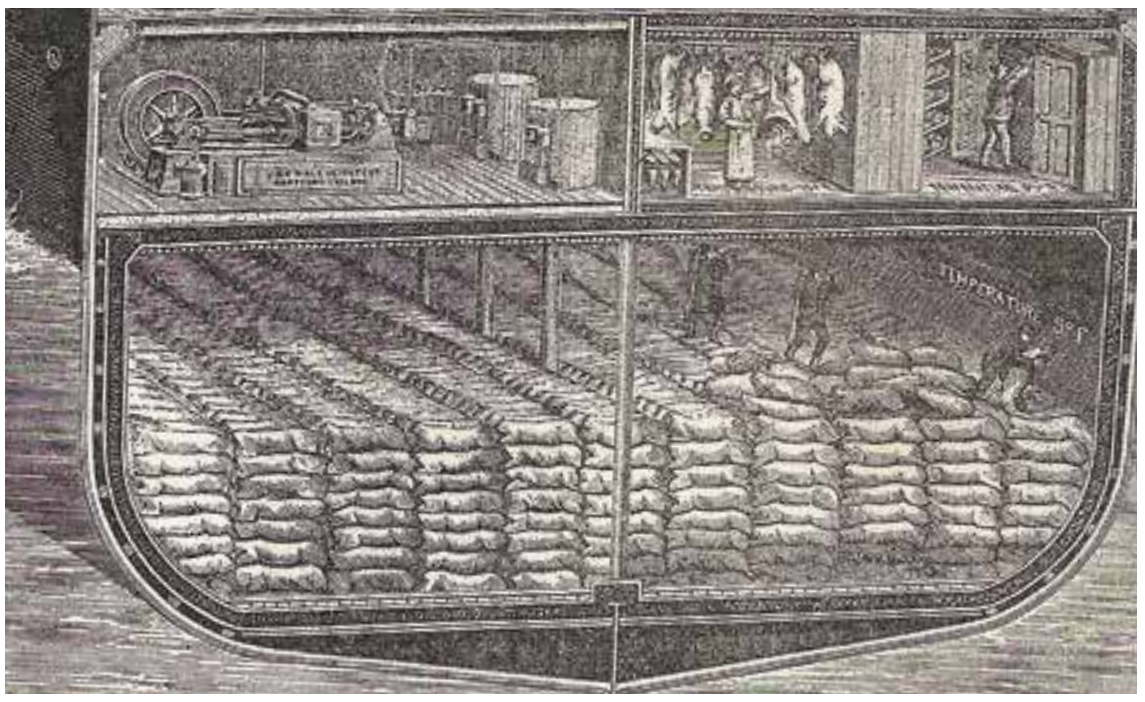
que la conservation de l'aliment se perpétue et que ses qualités prospèrent. Le vivant se rappelle à ses consommateurs, dans sa fragilité comme dans sa capacité à se reproduire, ici dans l'échange qui en assure la conservation collective.

Car conserver le vivant engage un ensemble complexe de règles et de normes, comme le montre Milena Estorniolo. Les Baniwa habitent les rives du Rio Içanaet ses affluents en Amazonie brésilienne, dans le Haut Rio Negro, une région réputée pour la pauvreté de ses eaux et de son sol. La pénurie de protéine animale marquant certaines périodes de l'année, la conservation des aliments joue un rôle central. Si cette société a développé et adopté des techniques de domestication et de conservation du manioc présentes en Amérique du Sud depuis 8000 ans, elle a refusé l'élevage de poissons proposé par une ONG pour résoudre la pénurie alimentaire. Lauteure montre que les techniques de conservation ne peuvent être saisies dans 


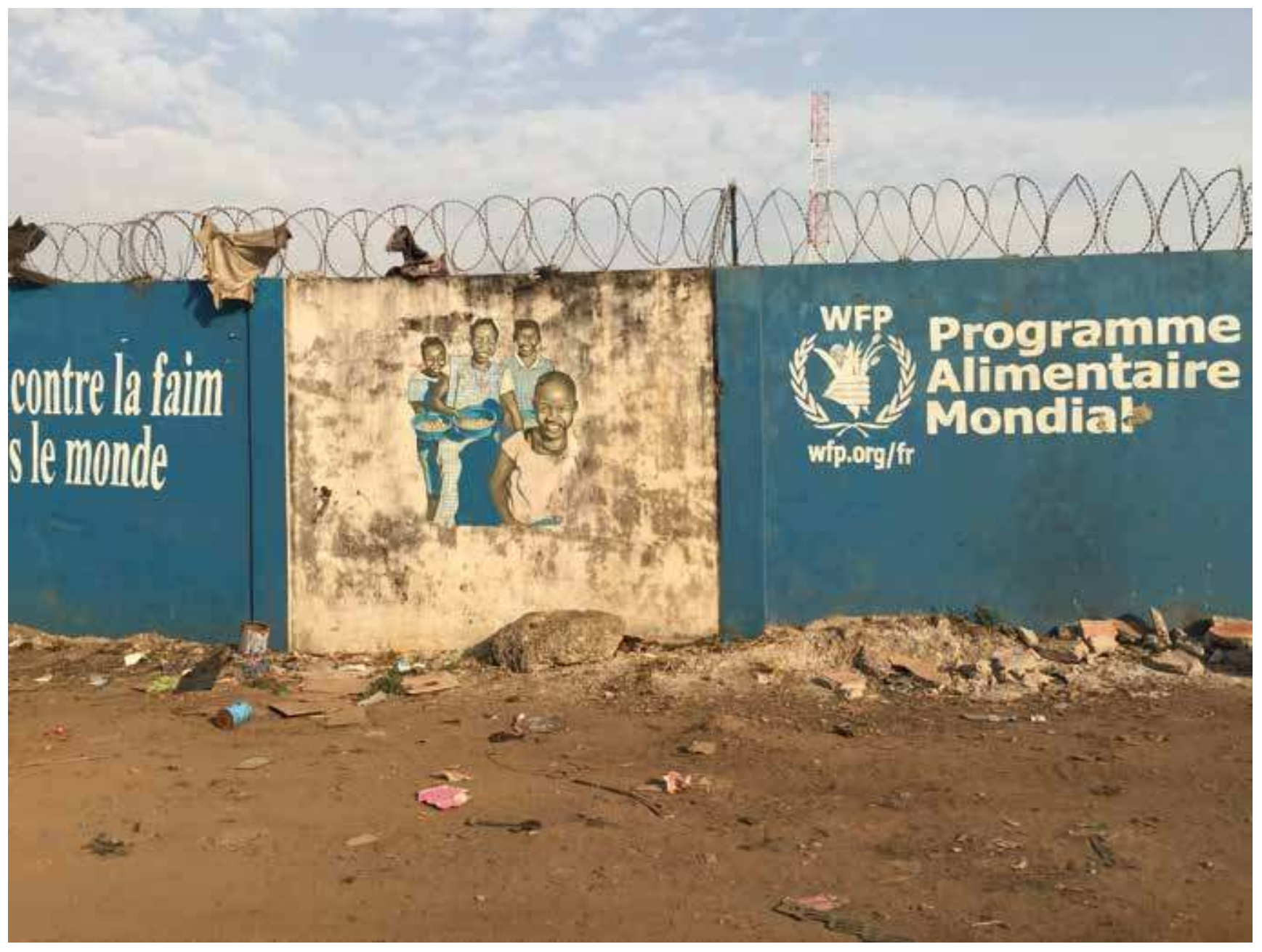

\section{Mur d'enceinte du site du Programme alimentaire mondiale onusien à Conakry, en Guinée, $\mathbf{2 0 1 6}$}

L'Organisation des Nations unies pour l'alimentation (FAO), le Programme alimentaire mondial (PAM) onusien, le Fonds international de développement agricole (FIDA), le Fonds des Nations unies pour l'enfance (UNICEF) et l'Organisation mondiale de la santé estiment qu'il y aurait eu 815 millions de personnes souffrant de sous-alimentation chronique dans le monde en 2016. Ces chiffres montrent une augmentation du nombre d'individus concernés par la faim dans le monde. 
leur complexité sans l'examen attentif, d'une part, des modalités de consommation, de partage, de distribution et de circulation de la nourriture (qui garantit, plutôt que le stockage, une certaine sécurité alimentaire à l'échelle d'une communauté) et, d'autre part, des relations qui lient une société à son environnement vivant non-humain.

Parfois, le partage peut également constituer une fin en soi, mobilisant des pratiques de requalification des aliments en limite de stockage. C'est le cas des Disco Soupes décrites par Jérémy Joncheray et Olivier Wathelet. À l'aide d'un dispositif mobile installé dans l'espace public, les organisateurs invitent les passants à se joindre à un moment de cuisine collective pour valoriser des rebuts alimentaires récoltés dans différents lieux de la ville. Plutôt que d'améliorer le stockage, il s'agit donc de valoriser les savoir-faire pour réduire le gaspillage alimentaire. Pratique à la fois marginale et fragile - absence de participants ou de produits à valoriser - elle constitue une tentative pour contrer les logiques sanitaires qui avaient, dans un premier temps, contraint la circulation de ces aliments. Car celle-ci, d'autant plus lorsqu'elle est large, pose la question des normes, une préoccupation qui semble traverser toutes les sociétés à travers le temps.

Le travail historique de Sandrine Robert montre le défi sanitaire, marchand et technique que représentait, au xive siècle, l'approvisionnement de Paris en poissons frais depuis les ports littoraux du Nord de la France. Comment pouvait-on acheter à Paris un poisson pêché au maximum 4,5 jours plus tôt - ce qui constituait la norme la plus stricte? Autour des chasse-marée qui approvisionnaient Paris, un dispositif régalien se met en place, encadrant la vente de cette marchandise fragile et très recherchée en raison des prescriptions religieuses en matière d'alimentation: entretien du réseau de transports, droits de ventes, contrôle de la marchandise... De ce fait, l'auteure montre que les normes sanitaires, et le contrôle des techniques de conservation qu'elles supposent, ne sont pas apparues avec la consommation de masse mais que les règles concernant ce que l'on appelle aujourd'hui les dates limites de vente et de consommation ont été définies dans un autre contexte sociopolitique, bien antérieur au nôtre.

La nécessité de «conserver à l'identique» associée à certaines situations de partage est également au cœur des dispositifs d'aide humanitaire eux aussi confrontés à l'exigence d'une circulation lointaine, dans des conditions matérielles, climatiques et sanitaires difficiles et instables. Alors que la poudre de lait se vend en vrac et peut être reconditionnée, comme le montre Samuel Pinaud dans la partie précédente, Jean-François Caremel, Raphaëlle Héron et Anne-Sophie Sauvegrain « déballent l'emballage» d'un aliment thérapeutique prêt à l'emploi (ATPE) auquel ont recours les organismes humanitaires. Suivant la production de cet ATPE, du laboratoire au terrain où ils sont distribués, les auteurs attirent l'attention sur son double emballage qui non seulement lui garantit son intégrité nutritionnelle et thérapeutique, mais lui permet de quitter les infrastructures médicales et de pénétrer les espaces domestiques où il peut être administré par les familles - et où son usage se trouve requalifié, souvent hors de toute indication médicale... Sur un terrain complètement différent, quoique pareillement médical puisqu'il s'agit de la prise en charge des prématurés, Céline Verguet s'intéresse également au basculement d'une denrée (le colostrum) du domaine alimentaire au domaine médical. Ici, 


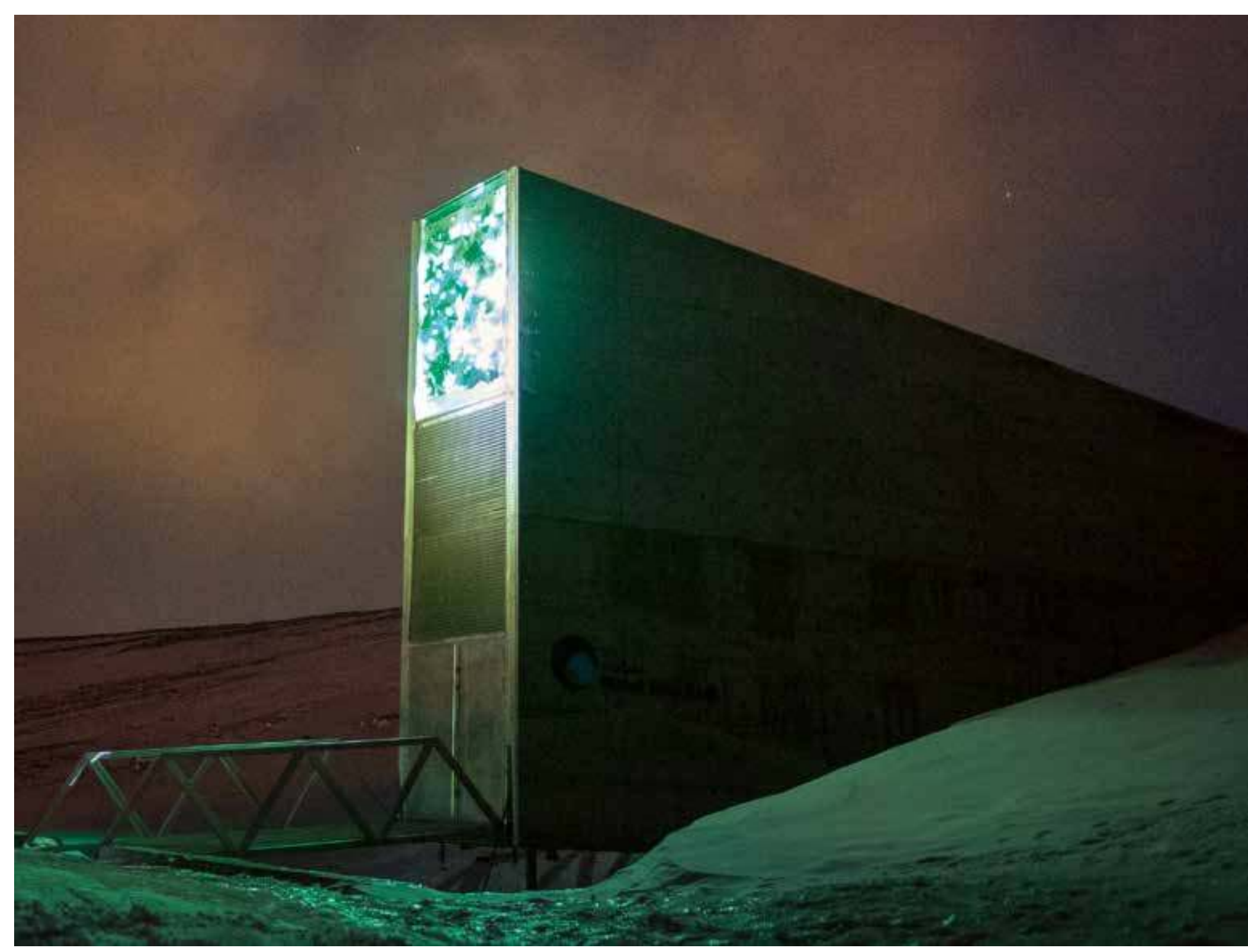

le partage «naturel», via l'allaitement, est impossible. Si les distances sont courtes (quelques étages), les enjeux sont énormes et c'est l'équipe médicale qui assure la transmission de la si précieuse mais fragile substance entre la mère et l'enfant. Les techniques de conservation du colostrum sont alors centrales pour maintenir les qualités nutritionnelles au long d'une chaîne opératoire, appelée à l'hôpital «protocole», le colostrum se délivrant aussi sur ordonnance. 


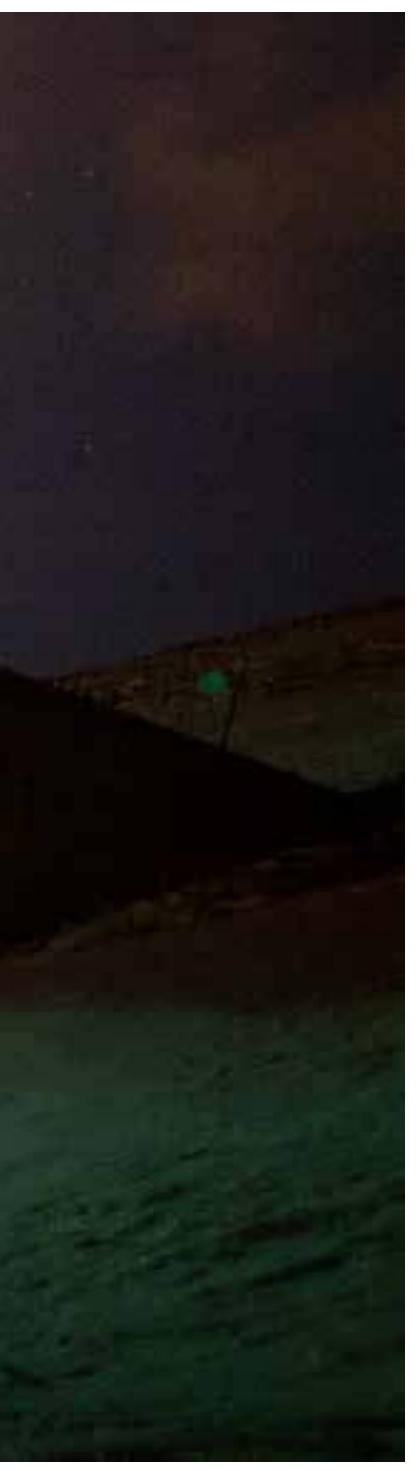

Cette question de la conservation à l'identique se pose d'une façon complètement différente dans le domaine de l'exploration spatiale où la durée de l'éloignement terrestre nécessite de recréer les pratiques alimentaires les plus quotidiennes qui contribuent au bien-être physique et psychologique des astronautes. Que l'astronaute français Thomas Pesquet célèbre son anniversaire avec quelques morceaux de Comté relève d'une longue négociation au sein de l'équipe logistique et des enjeux qui dépassent la simple anecdote ${ }^{7}$. Dans un environnement aussi contraint, les modalités de la conservation font l'objet de choix particulièrement réfléchis. Alain Maillet, responsable au Medes $(\mathrm{Cadmos})^{8}$, a accompagné scientifiquement et techniquement les pratiques alimentaires de la dernière expédition des Français sur l'ISS. Il revient sur l'histoire et les enjeux des techniques de conservation alimentaire dans les vols spatiaux pour faire ressembler le plus possible les repas en vol aux repas sur terre, le partage en étant une des composantes importantes. Cette situation fait écho au contexte des expéditions en itinérance sportive, étudiées par Éric Boutroy et Bénédicte Vignal qui en analysent les différences idéal-typiques. Ils montrent comment l'opposition, entre concevoir et optimiser ses rations alimentaires ou déléguer les tâches alimentaires à un cuisinier ou un accompagnateur, est imbriquée dans les chaînes opératoires alimentaires liées à la conservation. Les conditions extrêmes et les entreprises hors du commun (mouvements de populations, migrations, campagnes militaires, expéditions scientifiques, etc.) constituent à ce titre un laboratoire pour observer la contribution des techniques de conservation aux transformations et stabilisations des pratiques alimentaires et culinaires.

\section{Ne plus conserver?}

Qu'il s'agisse de conserver loin dans le temps ou de porter loin dans l'espace, la conservation donne à voir les soubassements techniques des cultures alimentaires et leurs cortèges d'enjeux tout à la fois nutritionnels, sociaux, familiaux, politiques, économiques et culturels... À l'instar des frigos entrouverts ici, le temps long de la conservation se marie au présent avec des enjeux très vifs, qui fait de ce thème un sujet éclairant pour penser les cultures alimentaires et leurs dynamiques. Surtout, il dépasse largement les limites de la simple transformation alimentaire. Dans ces différents articles, il apparaît au contraire que la conservation s'adosse à des pouvoirs et à des choix, possiblement mis en politique, et exercés aux différentes échelles de la vie sociale. Conserver ne va pas sans redistribuer, ce qui n'est autre qu'une façon de prévoir et de décider qui aura accès à quelle alimentation et quand. À ce
Le grain de blé est à la fois le produit d'une récolte et la ressource nécessaire au début d'un nouveau cycle de production. Il est donc nécessaire de protéger également les semences. La «Svalbard Global Seed Vault » en Scandinavie: littéralement la Chambre forte mondiale de graines du Svalbard a été officiellement ouverte en 2008 sur l'île norvégienne du Spitzberg. Elle est destinée à conserver dans un lieu sécurisé des graines de toutes les cultures vivrières de la planète et ainsi de préserver la diversité génétique. Elle est creusée à flanc de montagne à 120 mètres de profondeur: les 3 salles représentent $1500 \mathrm{~m}^{2}$ de stockage. Fin février 2018 , pour son dixième anniversaire, le nombre d'échantillons de semences d'espèces alimentaires qui y sont conservés dépasse le million. 
titre, vouloir augmenter la production agricole sans se poser la question de la redistribution ne constitue qu'une réponse insatisfaisante à la faim dans le monde.

Labondance relative de nos sociétés ne doit pas masquer un fait fondamental de l'évolution humaine: la capacité de conservation transforme les groupes sociaux jusque dans leurs humanités. Manifestement, ces processus restent à l'œuvre dans les différentes sociétés contemporaines et contribuent à les modeler. Car c'est dans la conservation que se résolvent également des tensions entre des projets de sociétés parfois antagonistes et que s'affirme la nécessité de la subsistance ou, quand elle semble acquise, celle de la valorisation d'une ressource rare ou originale. Les techniques de conservation contribuent donc à dessiner un ordre social parmi les mangeurs, au sein d'un espace social, politique et économique que nous explorons depuis une diversité de postes d'observation.

À ce titre, la conservation est un pivot porteur d'enjeux sociaux et d'une dimension politique forte: conserver permet de reprendre du pouvoir dans un nuancier très étalé, allant de l'extrême nécessité (Verguet, Carémel et al.) à la plus-value marchande (Fersing, Siniscalchi \& Zecchin, Pinaud), à l'importance du partage (Estorniolo ou Joncheray $\&$ Wathelet) ou simplement à l'attachement intime et personnel (Adamiec \& Savani). On peut dès lors réinterroger la non-conservation comme fait social: que dire de ceux pour qui cela constitue encore et toujours une lutte quotidienne dans l'accès à la nourriture? Ou quand, au contraire, l'absence de conservation domestique implique de vivre sans cesse dans une consommation située, en mobilité alimentaire permanente? Par leur violence symbolique et matérielle, ces marges questionnent la possibilité de réfléchir la production et la consommation hors de la conservation. Les pratiques décrites dans ces différents articles montrent pourtant que des choix sont possibles et que des modèles concurrents existent dans un même groupe social. Dès lors, la conservation alimentaire apparaît plus que jamais comme un objet bon à penser, mais également comme un levier bon pour agir et transformer les pratiques alimentaires contemporaines.

\section{Notes}

1. Le mot de conservation est utilisé dans cette introduction dans deux sens: d'abord comme synonyme de stockage; ensuite comme terme générique de «techniques de conservation», thématique de ce numéro. On parlera donc de l'ensemble des techniques qui permettent le stockage et la conservation de denrées alimentaires, soit au plus proche de leur état originaire, soit transformées.
2. Marseille, Mucem et Vieille Charité, les 26 et 27 janvier 2017. Celles-ci et la réalisation de ce numéro se sont inscrites dans le projet Open Food System soutenu par l'entreprise SEB, BPI France et Investissement d'Avenir et au sein duquel le laboratoire Dynamique Européenne, UMR 7367, avait en charge l'aspect sociologique et anthropologique de la recherche sous la direction scientifique de Nicoletta Diasio. Nous remercions particulièrement 
Nicoletta Diasio pour son soutien, ses conseils avisés et son regard vigilant et bienveillant tout au long de cette aventure scientifique collective.

3. http://www.gds.umontreal.ca/blog/portfolio/ les-oublies-des-fonds-de-frigo/.

4. Le rapport sur l'état de l'insécurité alimentaire dans le monde (SOFI), publié conjointement en septembre 2017 par la FAO, le PAM, le FIDA, l'Unicef et l'OMS, montre qu'une personne sur neuf environ souffre de sous-alimentation chronique (Maetz http://lafaimexpliquee.org).

\section{Iconographie}

Image d'ouverture. Épicière Ewé, vendant des cubes Maggi, des petits sacs de tomates, piments, combo, manioc... Marché de Ho (Volta, Ghana) 2007. Pour circuler et être éventuellement stockés, les aliments doivent être conditionnés. Ces techniques de conservation engagent des temporalités distinctes selon les aliments. (C) Frédéric Joulian.

\section{Références}

Cochoy, F. 2010 «Reconnecting marketing to "market-things" : How grocery equipments drove modern consumption» in L. Araujo, J. Finch \& H. Kjellberg dir. Reconnecting Marketing to Markets. Oxford: Oxford University Press: 29-49.

- 2017 «Making people buy and eat differently: Lessons from the modernisation of small independent grocery stores in the early twentieth century », Rev Agric Food Environ Stud (RAFES). [En ligne] : https://doi. org/10.1007/s41130-017-0046-5

Esquerre, L. \& A. Boltanski 2017 Enrichissement. Une critique de la marchandise. Paris: Gallimard.

Fine, B. \& E. Leopold 1993 The World of Consumption. Londres: Routledge.

Goody, J. 1985 Cuisines, cuisine et classes. Paris: Centre Georges Pompidou.

Howes, D. 1990 «Présentation. Les sensations discrètes de la bourgeoisie», Anthropologie et sociétés 14(2) : 5-12.
5. http://www.bondyblog.fr/201801051222/le-premier-frigo-solidaire-pour-lutter-contre-le-gaspillage-alimentaire-debarque-a-paris/\#. WndCbnjFYWV.

6. Produits labellisés par le mouvement Slow Food : https://slowfood.fr/.

7. http://next.liberation.fr/food/2017/05/23/2017-1odyssee-du-comte-dans-l-espace_1571703.

8. Centre d'Aide au Développement des activités en Micro-pesanteur et des Opérations Spatiales.

1. Photographie de presse ( ) Agence Rol.

2. Creative commons $\mathrm{CCO}$.

3. Détail d'un navire frigorifique. Antonin Rolet, 1913, Les conserves de légumes et de viandes des produits de la bassecour et de la laiterie. Paris: Lib. J. B. Baillière et Fils.

4. (C) Laetitia Atlani-Duault.

5. CC by 2.0 / Frode Bjørshol, 2015.

Karasati, H., Baker, K. \& F. Millerand 2010 «nfrastructure time: Long-term Matters in Collaborative Development », Computer Supported Cooperative Work $19(3-4): 377-415$.

Kujit, I. \& B. Finlayson 2009 «Evidence for food storage and predomestication granaries 11,000 years ago in the Jordan Valley», PNAS 106(27): 10966-10970.

Levi-Strauss, C. 1958 Anthropologie structurale. Paris: Plon. Mintz, S. 1985 Sweetness and Power: The Place of Sugar in Modern History. New York: Viking.

Régnier, F. 2004 L'exotisme culinaire. Essai sur la saveur de l'autre. Paris: PUF.

Testart, A. 1982 « The significance of food storage among hunter-gatherers: Residence patterns, population densities, and social inequalities», Current Anthropology 23: 523-537.

\section{Pour citer l'article}

Julien, M.-P., Wathelet, O. \& L. Dupré 2018 « Déjouer le temps et l'espace, reprendre du pouvoir», TechniquesECulture 69 «L temps des aliments», p. 12-27. 